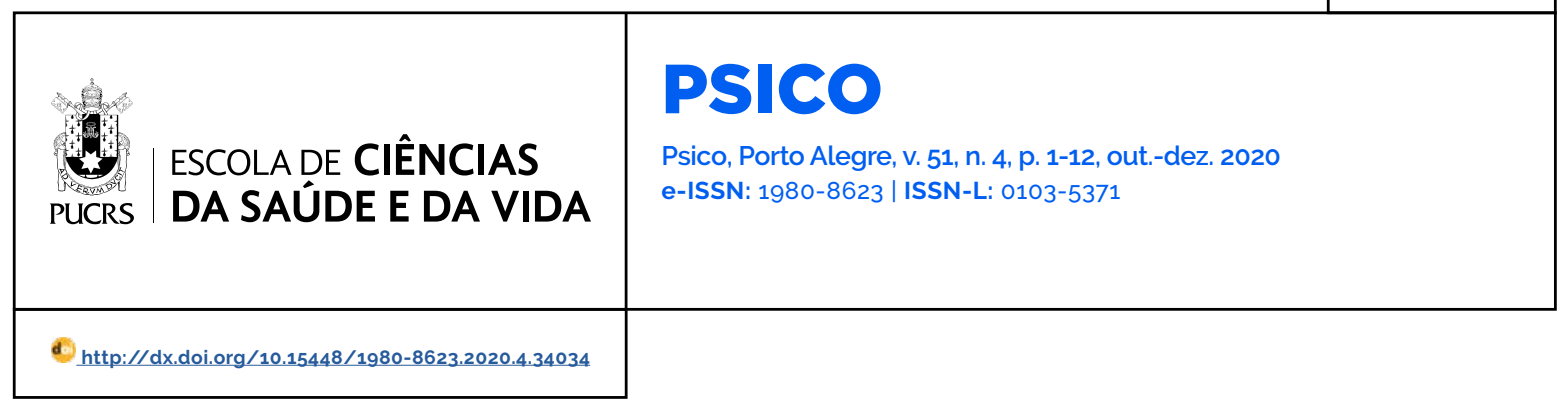

ARTIGO

\title{
Tratamento multicomponente para insônia infantil e seus efeitos nos padrões, hábitos e rotinas de sono
}

\author{
Multicomponent treatment for child insomnia and its effects on patterns, habits and \\ sleeping routines
}

Tratamiento multicomponente para el insomnio infantil y sus efectos sobre patrones, hábitos y rutinas para dormir

\section{Renatha El Rafihi- \\ Ferreira $^{1}$ \\ orcid.org/0000-0002-2212-4608 \\ rerafihi@usp.br}

\section{Maria Laura Nogueira \\ Pires $^{2}$}

orcid.org/0000-0001-8050-1187

laurapires@assis.unesp.br

\section{Edwiges Ferreira de}

Mattos Silvares ${ }^{1}$

orcid.org/0000-0002-4958-7549

efdmsilv@usp.br

Recebido em: 30 abr. 2019 Aprovado em: 14 set. 2020 Publicado em: 15 mar. 2021.

\section{(c) (1)}

Artigo está licenciado sob forma de uma licença Creative Commons Atribuicãa 4.0 Internacional.
Resumo: Problemas de sono são frequentes na infância e estão associados a problemas de comportamento. O objetivo deste estudo foi avaliar o efeito de um tratamento multicomponente para a insônia infantil nos padrões, rotina e hábitos de sono das crianças. Participaram 57 crianças com problemas comportamentais relacionados ao sono e suas mães. As mães receberam educação sobre o sono infantil e orientações sobre o estabelecimento de horários e rotinas para dormir e sobre o uso de técnicas de extinção e reforço positivo. Os dados foram avaliados antes e após a intervenção por diários de sono e pela Escala UNESP de Hábitos e Higiene do Sono - Versão Crianças. Nossos resultados apontaram que crianças com problemas de sono apresentam em sua maioria, hábitos e rotinas inadequadas antes de dormir e que uma intervenção que focasse na modificação destes hábitos, somada a estratégias de extinção para comportamentos inadequados e reforço positivo para comportamentos adequados no momento de dormir podem ter contribuido na melhora do sono. Espera-se que este trabalho possa estimular a produção de pesquisas nacionais sobre o tema, servindo como incentivo para o desenvolvimento de medidas preventivas e interventivas para os problemas de sono na infância.

Palavras-chave: sono, criança, rotina pré-sono, intervenção comportamental

Abstract: Sleep problems are common in childhood and are associated with behavioral problems. The aim of this study was to evaluate the effect of a multicomponent treatment for childhood insomnia on children's patterns, routine and sleep habits. 57 children with behavioral problems related to sleep and their mothers participated. The mothers were educated on their child's sleep, received guidance on the establishment of sleeping schedules and routines and on the use of extinction and positive reinforcement techniques. The data were evaluated before and after the intervention by sleep diaries and by the Escala UNESP de Hábitos e Higiene do Sono - Versão Crianças. Our results showed that children with sleep problems mostly present inadequate habits and routines before bedtime and that an intervention that focused on changing these habits, added to extinction strategies for inappropriate behaviors and positive reinforcement for adequate behaviors at bedtime may have contributed to improved sleep. It is hoped that this work can stimulate the production of national research on the subject, serving as an incentive for the development of preventive and interventional measures for childhood sleep problems.

Keywords: sleep, children, bedtime routine, behavioral intervention

Resumen: Los problemas de sueño son comunes en la infancia y están asociados con problemas de comportamiento. El objetivo de este estúdio fue evaluar el efecto de um tratamiento multicomponente para el insomnio infantil em los patrones, la rutina y los hábitos de sueño de los niños. Participaron 57 niños con 
problemas de conducta relacionados com el sueño y sus madres. Las madres recibieron educación sobre el sueño infantil y orientación sobre como estabelecer horarios y rutinas de sueño y sobre el uso de técnicas de extinción y refuerzo positivo. Los datos fueron evaluados antes y después de la intervención por diarios de sueño y por la Escala UNESP de Hábitos e Higiene do Sono - Versão Crianças. Nuestros resultados mostraron que los niños con problemas de sueño em su mayoria presentan hábitos y rutinas inadecuados antes de acostarse y que una intervención que se centróen cambiar estos hábitos, se agregó a las estrategias de extinción por conductas inapropiadas y refuerzo positivo para conductas adecuadas antes de acostarse puede haber contribuido a mejorar el sueño. Se espera que este trabajo pueda estimular la producción de investigación nacional sobre el tema, sirviendo como un incentivo para el desarrollo de medidas preventivas e intervencionistas para los problemas del sueño infantil. Palabras clave: sueño, infantil, rutina pre-sueña, intervención comportamental

O sono desempenha um papel fundamental na saúde, no desenvolvimento e no funcionamento diário das crianças (Allen, Howlett, Coulombe, \& Corkum, 2016). Contudo, um número considerável de crianças apresenta queixas de dificuldades de iniciar e manter o sono. Em crianças pequenas essas queixas são referidas como problemas no momento de dormir e despertares noturnos (Meltzer \& Mindell, 2014), com prevalência de 20\% a 30\% (Mindell \& Moore, 2014; Mindell, Kuhn, Lewin, Meltzer, \& Sadeh, 2006). De modo geral, a dificuldade para iniciar o sono e o despertar noturno ocorrem em $40 \%$ dos bebês recém-nascidos e em 20\% a $50 \%$ dos pré-escolares. Já a resistência a dormir é relatada em $15 \%$ a $27 \%$ das crianças em idade escolar (Durand, 2008; Owens, 2007). A prevalência no Brasil acompanha os registros internacionais. Um estudo nacional desenvolvido por Pires, Vilela e Câmara (2012) aponta que uma a cada duas crianças apresenta dificuldade para adormecer e uma a cada três desperta várias vezes durante a noite e se mostra sonolenta durante o dia.

A etiologia dos problemas de sono na infância é multifatorial e representa uma complexa interação entre comportamentos intrafamiliares, fatores culturais, fatores ambientais, fatores biológicos, circadianos e de desenvolvimento neurológico (Durand, 2008; Mindell et al., 2006). Os problemas no momento de dormir e os despertares noturnos em crianças podem ser vistos como similares ao modelo psicofisiológico da insônia em adultos, envolvendo fatores de predisposição, precipita- ção e perpetuação. Os fatores de predisposição referem-se a perturbações no ritmo circadiano e homeostático que formam o substrato neurobiológico sobre o qual esses problemas de sono são impostos. Os fatores precipitantes e perpetuantes são inúmeros, incluindo questões médicas, situações ambientais e relações parentais (Mindell et al., 2006; Reid, Huntley, \& Lewin, 2009).

Os problemas de sono em crianças podem ter um impacto negativo no humor, comportamento, desempenho acadêmico, aprendizado e consolidação da memória (Bolinger, Born, \& Zinke, 2018; Gruber et al., 2013). O comprometimento do sono na infância está associado à irritabilidade, agressividade, impulsividade, baixa tolerância à frustração, ansiedade, depressão, hiperatividade, labilidade emocional, desatenção e estresse familiar (Owens, 2007; Rafihi-Ferreira, Silvares, Pires, Assumpção-Junior, \& Moura, 2016). Considerando esses aspectos, intervir na quantidade e na qualidade do sono infantil é fundamental.

Entre as intervenções, destaca-se o uso do estabelecimento de rotinas pré-sono, a extinção para comportamentos incompativeis com o adormecer e o reforço positivo frente aos comportamentos pró-sono. Rotinas pré-sono envolvem um conjunto de atividades tranquilas que direcionam a criança para o momento de dormir. Os pais são orientados a estabelecer atividades relaxantes que devem ocorrer todas as noites em uma mesma ordem, em um período de 30 a 40 minutos. Essas atividades podem incluir, por exemplo, banho, livro de história, oração e cama. A escolha das atividades também deve respeitar a cultura familiar em que a criança está inserida. A ordem ideal das atividades deve mover-se progressivamente para o ambiente em que a criança deve dormir (Meltzer \& Mindell, 2011).

Para o estabelecimento de rotinas, os pais são orientados quanto à utilização da técnica do reforço positivo para ensinar à criança comportamentos apropriados em relação ao sono. A rotina pré-sono deve ser programada por meio de comportamentos antecedentes que indicam o momento de dormir, como escovar os dentes, vestir o pijama, ir para o quarto, deitar-se, escutar uma história ou cantiga e relaxar. No momento de estabelecer a 
rotina, é importante que a amamentação da criança ocorra no início da rotina, de forma a evitar que a criança adormeça mamando e associe o sono com o peito da mãe, com a mamadeira ou com o leite. Outro aspecto importante é evitar o uso de eletrônicos de 30 a 60 minutos antes do momento de dormir (Mindell \& Moore, 2014).

Os pais são orientados a reforçar (por meio de atenção, carinho, elogios, brinquedos etc.) os comportamentos adequados da criança (ficar quieto, não chorar, permanecer na cama) na rotina pré-sono e momentos antes de dormir (Didden, Sigafoos, \& Lancioni, 2011; Kuhn, 2011). Neste sentido, os pais são alertados a não reforçar comportamentos inadequados da criança (chorar, protestar). Os reforçadores devem ser contingentes aos comportamentos apropriados durante a noite (Didden et al., 2011; Kuhn, 2011). A Academia Americana de Medicina do Sono (2005) indica a técnica de extinção para o tratamento de problemas de sono na infância. Neste contexto, o objetivo da técnica é extinguir comportamentos aprendidos indesejáveis por meio da remoção dos reforços que mantêm o comportamento. Desta forma, os pais são orientados a ignorar os protestos (choro, birra) da criança no momento de dormir e quando desperta durante a noite. A extinção visa permitir que a criança desenvolva habilidades para adormecer sozinha, sem a ajuda dos pais (Hill, 2011).

Uma vez que a rotina pré-sono é estabelecida, os pais são orientados quanto ao estabelecimento de horários para dormir, de modo que, quando necessário, o horário de dormir é reprogramado gradualmente. O horário em que a criança está acostumada a dormir deve ser levado em conta, uma vez que colocar a criança em um horário muito anterior ao que ela está acostumada a dormir pode provocar resistência ao momento de dormir, bem como dificuldade de adormecer. Orienta-se colocar a criança na cama no horário em que ela está acostumada a dormir e depois reduzir gradualmente o horário para aquele desejado. Por exemplo, se a criança dorme às 21 horas, deve-se colocá-la às 21 horas na cama, e depois reduzir 15 minutos (20h45min) desse horário a cada 3-4 noites. Os horários devem ser consistentes, tanto nos dias úteis quanto nos finais de semana (Meltzer \& Mindell, 2011).

Estudos demonstraram que o tratamento multicomponente para o sono infantil com orientações parentais sobre estabelecimento de rotinas antes de dormir, extinção e reforço positivo levou à melhora do sono infantil (Mindell, Kuhn, Lewin, Meltzer, \& Sadeh, 2006; Mindell, Meltzer, Carskadon, \& Chervin, 2009; Mindell, Mond, Sadeh, Telofski, Kulkarni, \& Gunn, 2011). Considerando a importância da educação parental sobre o sono infantil tanto na prevenção como no tratamento de problemas de sono infantil, justifica-se estudos de intervenção sobe a temática. Embora haja um crescimento de investigações em problemas de sono infantil, os estudos na área ainda são incipientes, sobretudo no Brasil. O objetivo deste estudo foi avaliar o efeito de um tratamento multicomponente para a insônia infantil nos padrões, rotina e hábitos de sono das crianças.

\section{Método}

\section{Delineamento e aspectos éticos}

Trata-se de um estudo com delineamento pré-teste/pós-teste, em que os participantes foram submetidos a medidas anteriores e posteriores a um tratamento multicomponente. $\mathrm{O}$ estudo ocorreu em três etapas: pré-tratamento, tratamento e pós-tratamento.

Este estudo foi aprovado pelos Comitês de Ética em Pesquisa das universidades onde as intervenções foram realizadas e foi registrado no Registro Brasileiro de Ensaios Clínicos (RBR-4KXXD5).

\section{Participantes}

Participaram deste estudo 57 crianças com idade entre 1 e 5 anos, que preenchiam os seguintes critérios de inclusão: presença de problemas de ordem comportamental relacionados ao sono (insônia de associação, insônia por dificuldades de imposição de limites e a combinação entre elas). A criança devia apresentar alguma das seguintes caracteristicas citadas a seguir, em uma frequência de pelo menos três vezes na semana: (a) A criança demora cerca de 30 minutos ou mais para adormecer; (b) A criança resiste e/ou protesta ir para a cama; (c) A criança desperta 
durante a noite; e (d) A criança só dorme na presença dos pais. Crianças com comprometimento neurológico e/ ou sintomas psicóticos, conforme relatado pelos pais, foram excluídas.

Os dados sobre as crianças, fornecidos pelas mães, foram coletados como parte de um ensaio clínico randomizado que avaliou a eficácia de um programa de tratamento comportamental para problemas de sono dessas crianças (Rafihi-Ferreira, Pires, \& Silvares, 2019). O recrutamento ocorreu por meio de anúncios em jornais, bem como anúncios de rádio e TV. As caracteristicas da amostra das crianças e seus pais podem ser observadas na Tabela 1. A maior parte das crianças era do sexo masculino e tinha idade entre 12 e 42 meses. A idade das mães variou entre 23 e 46 anos, sendo que a maioria era casada e com ensino superior completo.

Tabela 1 - Caracteristicas sociodemográficas dos participantes

\begin{tabular}{|c|c|c|}
\hline & n (=57) & $\%$ \\
\hline \multicolumn{3}{|l|}{ Sexo da criança } \\
\hline Masculino & 34 & 60.0 \\
\hline Feminino & 23 & 40.0 \\
\hline \multicolumn{3}{|l|}{ Idade da criança } \\
\hline Média „Desvio padrão & \multicolumn{2}{|l|}{$2,36 \pm 1,32$} \\
\hline $12-42$ meses ( $1-31 / 2$ anos) & 44 & 77 \\
\hline $43-60$ meses ( $3 \sqrt{2}-5$ anos) & 13 & 23 \\
\hline \multicolumn{3}{|l|}{ Idade da mãe (anos) } \\
\hline Média \pm Desvio padrão & \multicolumn{2}{|c|}{$33,38 \pm 5,55$} \\
\hline Variação & \multicolumn{2}{|l|}{$23-46$} \\
\hline \multicolumn{3}{|l|}{ Estado civil } \\
\hline Casada & 46 & 80 \\
\hline Separada & 11 & 20 \\
\hline \multicolumn{3}{|l|}{ Escolaridade da mãe } \\
\hline Ensino médio & 22 & 39 \\
\hline Ensino superior & 35 & 61 \\
\hline \multicolumn{3}{|l|}{ Estrato social } \\
\hline Alta & 19 & 33 \\
\hline Média & 30 & 53 \\
\hline Baixa & 08 & 14 \\
\hline
\end{tabular}

Nota: Estrato social foi definido pelos itens do Critério de Classificação Econômica do Brasil (ABEP, 2012), que resulta em um escore com classificações compativeis com classificações baixa, média e alta.

\section{Procedimento}

Os dados foram coletados em três clínicas-escolas de Psicologia de universidades públicas no periodo de 2012 a 2014. Os pais que se inscreveram para participar da intervenção para problemas de sono infantil foram informados sobre os objetivos, métodos e fases do estudo, e assegurados de que os dados fornecidos por eles seriam tratados de forma anônima e confidencial e, então, assinaram o Termo de Consentimento Livre e Esclarecido. Os cuidadores forneceram informações sobre os hábitos e rotinas da criança, respondendo ao instrumento, em sessões que duraram aproximadamente 30 minutos. A pesquisadora forneceu explicações sobre a conclusão do instrumento e perguntou se os pais preferiam responder com ou sem assistência. Mesmo nos casos em que o cuidador optou por responder sem assistência, a pesquisadora permaneceu na sala para auxiliar se houvesse alguma dúvida.

A primeira avaliação foi realizada uma semana antes do tratamento multicomponente e a segunda avaliação, uma semana após a conclusão do tratamento. O intervalo entre as avaliações foi de sete semanas.

\section{Tratamento multicomponente}

O tratamento multicomponente ocorreu por meio de orientação parental em cinco sessões em um periodo de sete semanas. As sessões foram individuais com a mãe de cada criança e foram conduzidas pela primeira autora que, na época do estudo, era estudante de doutorado em Psicologia Clínica. A pesquisadora recebia semanalmente supervisão de suas professoras/orientadoras em relação à psicologia clínica infantil e psicologia do sono. Durante as sessões, as mães receberam educação parental sobre o sono e o desenvolvimento infantil, com informações acerca das mudanças necessárias no sono das crianças nos primeiros anos de vida. Além disso foram orientadas quanto ao estabelecimento de horários e rotinas para dormir, com informações sobre o padrão consistente de sono e o horário adequado à idade para dormire acordar. Explicações sobre a manutenção dos problemas de sono, de acordo com as consequências 
posteriores ao comportamento, foram abordadas durante as sessões. A utilização do reforço positivo e a extinção de atividades prévias ao sono e a higiene do sono também foram discutidas nas sessões de orientação parental.

\section{Instrumentos}

Diário de Sono. Os diários de sono foram elaborados pelas autoras com o objetivo de registrar os horários em que o participante dormiu e despertou, estimativa de quanto tempo demorou a adormecer e o número dos despertares ao longo da noite. Os diários foram realizados durante todo o periodo de estudo.

Escala UNESP de Hábitos e Higiene do Sono - Versão Crianças. Trata-se de um instrumento com questões fechadas que buscam avaliar as características do sono de crianças, respondidas pelo cuidador, elaborado por Pires et al. (2012). A escala é composta de questões iniciais sobre os horários habituais de sono da criança e de uma seção chamada Hábitos de Higiene do Sono, com 16 questões (pontuadas em uma escala de frequência) que avaliam: indicadores sobre rotina e hábitos positivos e negativos do sono (conjunto de atividades que precedem o horário de dormir); alerta fisiológico (conjunto de hábitos diurnos que promoveriam estado de excitação ou desconforto físico antes do horário de dormir); aspecto cognitivo/emocional (engloba indicadores de regulação e bem-estar emocional pré-sono); e conforto do ambiente de dormir. A escala ainda conta com itens suplementares que avaliam a presença de dificuldades para dormir e sonolência diurna (anotados em uma escala de frequência). A confiabilidade da seção Hábitos de Higiene do Sono foi estimada por meio do coeficiente Alfa de Cronbach em uma amostra composta por pré-escolares e escolares, resultando em um valor de 0,68, o que assegura sua confiabilidade.

\section{Análise dos dados}

O Statistical Package for the Social Sciences (SPSS), versão 22.0, foi utilizado para análise estatística. Foram realizadas análises descritivas e inferenciais. Para caracterizar as rotinas, os hábitos e os padrões de sono foram calculadas proporções, médias e desvio-padrão. Os dados dos diários de sono foram analisados e comparados considerando o período pré e pós-tratamento. Comparações entre os momentos pré e pós foram realizadas por meio do teste $t$ para amostras pareadas, seguido do cálculo de magnitude do efeito. A magnitude do efeito, expressa como $d$, foi estimada como a diferença entre as médias dos grupos pré e pós-intervenção dividida pelo desvio-padrão comum. Convencionalmente, um valor de $d=0,20$ representa uma magnitude de efeito pequeno, $d=0,50$ indica magnitude média e $d=0,80$ indica uma magnitude de efeito elevada (Cohen, 1988).

Para comparar a diferença de proporção em relação às rotinas e os hábitos de sono nos momentos pré e pós-tratamento foi realizado o teste não paramétrico McNemar, seguido pelo cálculo do tamanho do efeito $\mathrm{g}$ de Cohen. Um valor de $\mathrm{g}$ de Cohen=0,05 $-<0.15$ representa um efeito pequeno, $\mathrm{g}=0,15-<0,25$ um efeito médio e $\mathrm{g} \geq$ 0,25 um efeito elevado (Cohen, 1988).

\section{Resultados}

Os resultados foram divididos em: (a) Padrões do sono das crianças e (b) Rotinas e hábitos das crianças relacionados ao sono.

\section{Padrões de sono das crianças}

A Tabela 2 apresenta as variáveis de sono das crianças avaliadas por diários de sono no período pré e pós de todos os participantes submetidos à intervenção. Como pode ser observado na Tabela 2, após a intervenção as crianças passaram a dormir mais cedo, a adormecer mais rápido, a ter menos despertares por noite e a terem maior duração do sono. Para esses fatores, a análise estatística demonstrou valores $d$ de médios a altos, demonstrando que há uma diferença substancial entre as médias dos dois momentos (pré e pós). 
Tabela 2 - Padrões do sono das crianças avaliadas por Diário de Sono

\begin{tabular}{lccccccc}
\hline \multicolumn{1}{c}{ VARIÁVEIS } & \multicolumn{2}{c}{ PRÉ (n=57) } & \multicolumn{2}{c}{ Pós (n=57) } & & d \\
\hline & M & DP & M & DP & t & d \\
\hline Horário de deitar (decimal) & 22.66 & 1.64 & 21.81 & 1.23 & $3.13^{*}$ & 0.58 \\
Horário de levantar (decimal) & 8.26 & 1.16 & 8.07 & 1.05 & 0.91 & - \\
Latência (minutos) & 50.14 & 31.84 & 18.18 & 18.04 & $6.59^{*}$ & 1.23 \\
Despertares/noite & 2.38 & 1.31 & 0.17 & 0.46 & $12.01^{*}$ & 2.25 \\
Duração total sono (horas/decimal) & 9.54 & 1.22 & 10.22 & 1.08 & $3.15^{*}$ & 0.59 \\
\hline
\end{tabular}
${ }^{*} \mathrm{p}<0.05$

\section{Rotina e hábitos das crianças relacionados ao sono}

A Tabela 3 e a Figura 1 apresentam a comparação entre a rotina e os hábitos dos participantes antes e depois de serem submetidos ao tratamento multicomponente. Como pode ser visto na Tabela 3 e na Figura 1, segundo o relato das mães na fase pré-tratamento, a maioria das crianças tinha rotina pré-sono, que para muitos, incluía hábitos positivos como o banho/escovar os dentes. No entanto, para grande parte das crianças, a rotina era composta também por hábitos negativos, como assistir TV e/ou jogar video game, praticar atividades vigorosas e adormecer mamando. Poucas crianças dormiam no horário estabelecido e independentemente. Grande parte das crianças adormecia em outro lugar antes de ir ou ser colocada na cama, não dormia sozinha, despertava frequentemente à noite e solicitava atenção ou ia para a cama dos pais. Além disso, um número considerável de crianças apresentava emoções fortes (raiva, medo ansiedade) momentos antes de dormir e acordava com aparência cansada pela manhã.

Observa-se na Tabela 3 e na Figura 1 que, após o tratamento multicomponente, houve mudanças significativas, com o aumento de hábitos e de rotinas positivas (orações, histórias, conversar sobre o dia, adormecer de forma independente e dormir e acordar no horário estabelecido) e a redução de hábitos e de rotinas negativas (adormecer mamando, atividades vigorosas, TV/videogame, emoções fortes, compartilhar a cama, despertares com solicitações de atenção e a aparência de cansaço pela manhã).

Nota-se uma diferença estatisticamente significativa nos periodos pré e pós-tratamento em relação à proporção das crianças, indicando que houve mudanças na maioria dos hábitos e das rotinas, com exceção do lanche leve, da música e do embalo após o tratamento multicomponente. $\mathrm{O}$ cálculo de magnitude de efeito expresso como g de Cohen demonstrou valores elevados representando uma diferença substancial entre os momentos pré e pós-intervenção para quase todas as variáveis.

Tabela 3 - Rotina e hábitos das crianças relacionados ao sono

\begin{tabular}{|c|c|c|c|c|c|c|}
\hline & \multicolumn{2}{|c|}{ PRÉ (n=57) } & \multicolumn{2}{|c|}{ PÓS (n=57) } & \multirow[t]{2}{*}{$p$} & \multirow[t]{2}{*}{ g } \\
\hline & $\mathbf{n}$ & $\%$ & $\mathbf{n}$ & $\%$ & & \\
\hline Rotina Pré-Sono & 46 & 81 & 55 & 96 & $<0,05$ & 0,50 \\
\hline \multicolumn{7}{|l|}{ Hábitos e rotina positivos } \\
\hline Banho / escovar os dentes & 30 & 53 & 47 & 83 & $<0,05$ & 0,50 \\
\hline Acorda no horário & 26 & 46 & 42 & 74 & $<0,05$ & 0,29 \\
\hline Dorme no horário & 16 & 28 & 37 & 65 & $<0,05$ & 0,50 \\
\hline
\end{tabular}




\begin{tabular}{|c|c|c|c|c|c|c|}
\hline & \multicolumn{2}{|c|}{ PRÉ (n=57) } & \multicolumn{2}{|c|}{ PÓS $(n=57)$} & \multirow[t]{2}{*}{ p } & \multirow[t]{2}{*}{ g } \\
\hline & $\mathbf{n}$ & $\%$ & $\mathbf{n}$ & $\%$ & & \\
\hline Orações & 14 & 25 & 20 & 35 & $<0,05$ & 0,50 \\
\hline Histórias & 11 & 19 & 18 & 32 & $<0,05$ & 0,50 \\
\hline Conversar sobre o dia & 6 & 10 & 17 & 30 & $<0,05$ & 0,42 \\
\hline Lanche leve & 5 & 9 & 9 & 16 & n.s. & --- \\
\hline Música relaxante & 3 & 5 & 4 & 7 & n.s. & --- \\
\hline Dorme independente & 2 & 4 & 41 & 72 & $<0,05$ & 0,50 \\
\hline \multicolumn{7}{|l|}{ Hábitos e rotinas negativos } \\
\hline Desperta e pede atenção & 50 & 88 & 5 & 9 & $<0,05$ & 0,50 \\
\hline Eletrônicos (TV, videogame) & 48 & 84 & 9 & 16 & $<0,05$ & 0,48 \\
\hline Vai para cama dos pais & 45 & 79 & 2 & 4 & $<0,05$ & 0,50 \\
\hline Atividades vigorosas & 45 & 79 & 8 & 14 & $<0,05$ & 0,50 \\
\hline Adormece em outro lugar & 36 & 63 & 4 & 7 & $<0,05$ & 0,50 \\
\hline Mamadeira / amamentação & 30 & 53 & 14 & 25 & $<0,05$ & 0,50 \\
\hline Divide a cama & 24 & 42 & 2 & 4 & $<0,05$ & 0,50 \\
\hline Emoções fortes & 23 & 40 & 3 & 5 & $<0,05$ & 0,50 \\
\hline Acorda cansada & 23 & 40 & 3 & 5 & $<0,05$ & 0,50 \\
\hline Embalo & 6 & 10 & 4 & 7 & n.s. & --- \\
\hline
\end{tabular}



Hábitos/rotina positivos

Hábitos/rotina negativos

Figura 1 - Rotina e hábitos das crianças antes e depois do tratamento Nota: * $p<0.05:{ }^{*} g \geq 0,25$

\section{Discussão}

Este estudo foi o primeiro no Brasil a identificar e descrever a rotina e os hábitos de crianças pré-escolares típicas com problemas de sono antes e após um tratamento multicomponente para insônia infantil. Os resultados desse estudo sugerem que o tratamento multicomponente, com estabelecimento de rotinas, extinção de 
comportamentos incompatíveis com o sono e reforço de comportamentos pré-sono é eficaz para problemas no momento de dormir e despertares noturnos em crianças com idade entre um e cinco anos. Foram encontradas mudanças nos hábitos, nas rotinas, nos horários e nos padrões de sono, avaliadas por medidas parentais, das crianças após suas mães receberem orientações para o manejo da insônia infantil.

Quanto aos padrões de sono, observou-se que uma quantidade significativa de crianças tinha horários tardios de ir para a cama, demorava para adormecer, despertava à noite e dormia menos de dez horas por noite. Esses padrões apontam presença de problemas de sono. Embora as horas diárias recomendadas de sono para pré-escolares sejam de 10 a 13 horas (Hirshkowitz et al., 2015), nossos resultados apontam que muitas crianças não dormem o suficiente. $\mathrm{A}$ obtenção de quantidades adequadas de sono é importante para o desenvolvimento social, emocional, comportamental e acadêmico das crianças (Scharf, Demmer, Silver, \& Stein, 2013). Em crianças pré-escolares, a duração do sono tem um efeito ainda mais importante (Brambilla et al., 2017; Chaput et al., 2017; Gruber et al., 2014; Khan et al., 2015), uma vez que está intrinsecamente associado a fatores como crescimento, desenvolvimento cognitivo e comportamental, maturação do sistema nervoso central e manutenção do balanço energético (Chaput et al., 2017; Gruber et al., 2014; Moreira \& Pradella-Hallinan, 2017; Short et al., 2018). A demora para adormecer poderia ser decorrente da falta de rotina pré-estabelecida e de hábitos incompativeis com o processo de adormecer. De fato, Mindell et al. (2009) já apontou que crianças com ausência de uma rotina consistente de dormir apresentam menor quantidade de sono. Outro aspecto a ser considerado são os horários de ir para a cama, o estudo de Crabtree, Korhonenb, Montgomery-Downs, Jones, O'Brien e Gozala (2005) aponta que crianças pequenas e pré-escolares dormem menos quantidade de horas de sono devido ao horário tardio de dormir, depois das 21 horas. Na pesquisa de Mindell, et al. (2009), dormir depois das 21 horas teve associação com maior tempo para adormecer e menor tempo total de sono.

De acordo com os resultados, após a intervenção as crianças passaram a dormir mais cedo, a dormir em menor frequência com seus pais, além de apresentarem melhora nos padrões de sono, caracterizada por menor tempo para adormecer independentemente e sem protestar, maior tempo total de sono e redução nos despertares noturnos. Apesar de as crianças passarem a dormir mais cedo, dormir um pouco antes das 22 horas ainda é considerado tarde para crianças na faixa etária pré-escolar. Observou-se que, depois do tratamento, a média do tempo total do sono das crianças aumentou, bem como aumentou o número de crianças que passaram a dormir mais do que dez horas por noite. O tempo total de sono da criança varia de acordo com a idade. Segundo a Foundation National Sleep (2016), para as idades entre um e três anos a quantidade total de sono é de 12 a 14 horas, e para crianças de três a cinco anos, de 11 a 13 horas. Ao considerar o sono noturno, de acordo com estudiosos da área (Ferber, 2006; Iglowstein, Oskar, Jenni, Molinari, \& Largo, 2003; Sheldon, 2014), a duração do sono noturno para essa faixa etária varia aproximadamente entre 9 e 11 horas.

Em comparação com outro estudo brasileiro (Pires et al., 2012), que indica que cerca de 60\% das crianças pré-escolares dormem de 9 a 11 horas, a quantidade de horas de sono na etapa pré-tratamento era baixa e, após a intervenção, ficou próxima e corroborando os dados do estudo de Pires et al. (2012). Tais alterações podem ser decorrentes das orientações que ressaltaram a importância de as crianças terem seus próprios hábitos e rotinas e não compartilharem os horários e rotinas do adulto, o que pode ter contribuido para as crianças irem dormir mais cedo e, consequentemente, terem maior duração do sono.

Quanto às demais variáveis do sono, avaliadas por medidas parentais, houve redução na latência para início do sono e número de despertares noturnos após a intervenção. A maior parte das crianças passou a adormecer em menos de 30 minutos, resistir menos à cama, despertar 
menos, além de grande parte passar a dormir independentemente, isto é, aquelas que dormiam apenas na presença dos pais ou compartilhavam a cama por dificuldade em adormecer passaram a dormir sem a necessidade de o cuidador estar presente. Os resultados do presente estudo demonstraram tamanhos de efeito significativos, em particular no que diz respeito aos padrões de sono como latência de início do sono e despertares noturnos, corroborando os resultados de pesquisas que indicaram que crianças que foram submetidas a uma intervenção que incluía componentes destinados a promover a capacidade da criança se auto-acomodar e adormecer de forma independente, como a extinção, levou a melhorias significativas na qualidade do sono, como redução na latência do sono (Blunden, 2011) e despertar noturno (Blunden, 2011; Moore, Friman, Fruzzetti, \& Macaleese, 2007; Seymour, Bayfield, Brock, \& During, 1983). No presente estudo, a extinção pode ter desempenhado um papel-chave na redução de despertares e um aumento da independência das crianças na hora de dormir. Ao reduzir a presença parental antes de dormir, as crianças tiveram a oportunidade desenvolver habilidades de autorregulação que são importantes para aprender a dormir de forma independente. De acordo com Thomas, Moore e Mindell (2014), o sucesso nos resultados da extinção ocorre porque a técnica alcança o objetivo de promover o desenvolvimento de habilidades de auto-acomodação na criança. De fato, a revisão realizada por Allen et al. (2016) apoia firmemente as recomendações que incentivam independência na hora de dormir, a fim de quebrar associações negativas de início do sono.

A rotina do sono tem o objetivo de desenvolver associações entre hábitos positivos, isto é, um conjunto de atividades tranquilas que direcionam a criança para o momento de dormir e o rápido adormecer. Na presente pesquisa, apesar da maioria das mães relatarem que suas crianças já apresentavam uma rotina pré-sono, essa era composta por poucas atividades, na maioria dos casos, apenas composta por banho e mamadeira. Após a intervenção, os participantes passaram a ter uma rotina mais consistente e deixaram de adormecer mamando. As crianças que continuaram mamando passaram a mamar no início da rotina e não mais no final, como na etapa inicial. Adormecer mamando fortalece associações inapropriadas para o sono; uma vez que esse componente não esteja mais associado ao adormecer, a criança necessita desenvolver habilidades para dormir independentemente. Além disso, com a intervenção, mais crianças passaram a incluir em sua rotina o banho/escovar os dentes, conversar sobre o dia e escutar histórias antes de dormir. Segundo Mindell, Telofski, Wiegand e Kurtz (2009), o banho como componente da rotina pré-sono pode afetar a temperatura corporal e, consequentemente, melhorar o sono. A inclusão do banho na rotina foi relatada no estudo de Mindell et al. (2009), apresentando resultados favoráveis. A inclusão de histórias antes de dormir, além de ser um componente positivo de associação para início de sono, incentiva uma prática cultural importante para a educação da criança. Esse estudo corrobora estudos anteriores (Adam \& Rickert, 1989; Mindell et al., 2009a; Mindell, 2009), que já relataram a importância do estabelecimento de rotinas na melhora dos padrões de sono das crianças.

Assistir à televisão/jogar videogame era um hábito bastante frequente nas crianças da presente amostra durante a etapa de avaliação. Contudo, quando as mães receberam orientação parental, grande parte das crianças deixou de assistir à televisão ou jogar videogame no momento de dormir. Ficar exposto à televisão antes de dormir pode interferir nos padrões de sono, muitas vezes, deixando a criança alerta e ativa, uma vez que a exposição à luz da tela da tevê tem associação com baixa concentração de melatonina (Salti et al., 2006), que interfere no adormecer. $\mathrm{O}$ estudo de Mistry, Minkovitz, Strobino e Borzekowski (2007), que teve como objetivo investigar a relação entre exposição à tevê nos primeiros anos de vida e problemas de comportamento aos cinco anos de idade, indicou que o comportamento de assistir à televisão por mais de duas horas ao dia na primeira infância e na idade pré-escolar está associado com maio- 
res problemas de sono, problemas de atenção, comportamento agressivo e comportamentos de externalização, avaliados pelo Child Behavior Checklist. Esse estudo indicou ainda que a presença do televisor no quarto da criança com 5.5 anos de idade foi, em geral, associada a problemas comportamentais e redução de habilidades sociais. Considerando os prejuizos da exposição à televisão, a presente intervenção conseguiu resultados benéficos, uma vez que houve decréscimo desse hábito após as mães participaram da intervenção.

Não dormir sozinho(a) teve destaque na presente amostra, com a maioria das crianças pegando no sono em outro local antes de ser levada para a cama. Sobre esse aspecto, estudiosos (Ferber, 2006; Sadeh, Tikotzky \& Scher, 2010) apontam que, independentemente da cultura, alguns pais ficam mais tranquilos ao dormir junto com suas crianças durante à noite por estarem por perto caso elas precisem de ajuda. Em contrapartida, os autores indicam que algumas desvantagens podem estar associadas a essa prática. Ao dividir a cama, pais e filhos podem despertar mais, demorar mais para dormir, além de as crianças dormirem tarde em decorrência da rotina parental. Mindell, Sadeh, Kohyama e How (2010) indicam que o compartilhamento da cama está associado com maior número de despertares e menor quantidade de horas de sono. Além disso, a criança pode associar o início do sono com a presença dos pais e só conseguir adormecer na presença destes, tendo dificuldade em dormir sozinha. Neste estudo, essa prática foi reduzida após a implementação da intervenção, o que sugere que a presença do cuidador poderia estar associada com o início do sono e que o tratamento foi efetivo para a criança dormir independentemente. Sadeh et al. (2010) enfatizam que dormir independentemente, ou seja, autoacomodar-se é o componente principal para o melhor desenvolvimento dos padrões de sono, resultando em decréscimo de problemas de sono e em aumento da consolidação do sono. Ao deixar de compartilhar a cama e também as rotinas dos adultos, as crianças passam a dormir mais cedo, despertar menos e ter maior quantidade de sono noturno (Mindell et al., 2010; Sadeh et al., 2010). Na presente pesquisa, com as orientações, as mães puderam compreender a importância de a criança desenvolver habilidades para adormecer independentemente, e muitas crianças, depois disso, deixaram de dormir compartilhando a cama.

Conhecer detalhadamente os hábitos e as rotinas de sono da criança com dificuldades com o sono é de extrema importância para o desenvolvimento de medidas preventivas e interventivas. Estudo anterior, realizado por Mindell et al. (2009) já reportou a associação entre hábitos inadequados (horário tardio de ir para a cama, presença parental no momento de dormir e TV no quarto) e má qualidade de sono. No entanto, este é o primeiro estudo brasileiro que demonstra quais os hábitos e as rotinas de crianças com problemas de sono e a mudança dos hábitos pós-intervenção. Os resultados obtidos mostraram valores de $\mathrm{g}$ de Cohen elevados para quase todas as variáveis de rotina e de hábitos de sono demonstrando que o tratamento multicomponente teve efeito semelhante sobre elas.

No entanto, considerando que a presente intervenção foi composta por vários elementos (estabelecimento de rotinas, extinção e reforço positivo), a partir do delineamento deste estudo não é possivel determinar qual elemento da intervenção foi responsável pela melhora dos padrões de sono das crianças. Supomos que os elementos terapêuticos em conjunto exerceram influência no desfecho positivo do estudo. Para avaliar qual elemento terapêutico é mais eficaz na melhora dos padrões de sono, futuros estudos devem comparar cada elemento como monoterapia e ainda comparar diferentes monoterapias com uma intervenção multiterapêutica para de fato avaliar se o elemento terapêutico isolado ou combinado é mais eficaz.

Concluindo, este estudo demonstrou que crianças com problemas de sono apresentam, em sua maioria, hábitos e rotinas inadequadas antes de dormir e que um tratamento multicomponente com educação parental para o sono com estabelecimento de rotinas, extinção e reforço positivo, pode contribuir para a melhora 
do sono infantil. Considerando o impacto que os problemas de sono podem causar na qualidade de vida da criança, justifica-se os estudos nessa temática. Além disso, o estudo do sono infantil, sobretudo no Brasil, ainda é incipiente, tendo uma escassez de publicações na área. Espera-se que este trabalho possa estimular a produção de pesquisas nacionais sobre o tema, servindo como incentivo para o desenvolvimento de medidas preventivas e interventivas para os problemas de sono na infância.

\section{Referências}

American Academy of Sleep Medicine. (2005). International classification of sleep disorders: diagnostic and coding manual (2 ed.). Westchester, IL: American Academy of Sleep Medicine.

Adams, L. A. \& Rickert, V. I. (1989). Reducing bedtime tantrums: Comparison between Positive routines and Graduated Extinction. Pediatrics, 84(1), 756-761.

Allen, S. L., Howlett, M. D., Coulombe, J. A., \& Corkum, P. V. (2016). ABCs of Sleeping: A review of the evidence behind pediatric sleep practice recommendations. Sleep Medicine Reviews, 29(1), 1-14. https://doi.org/ 10.1016/j.smrv.2015.08.006

Blunden, S. (2011). Behavioural treatments to encourage solo sleeping in pre-school children: an alternative to controlled crying. Journal of Child Health Care, 15, 107-117.

Bolinger, E., Born, J., \& Zinke, K. (2018). Sleep divergently affects cognitive and automatic emotional response in children. Neuropsychologia, 117(1), 84-91. https://doi. org/10.1016/j.neuropsychologia.2018.05.015

Brambilla, P., Giussani, M., Pasinato, A., Venturelli, L., Privitera, F., ... Chiappini, E. (2017). Sleep habits and pattern in 1-14 years old children and relationship with video devices use and evening and night child activities. Italian Journal of Pediatrics, 43(1). https://doi. org/10.1186/s13052-016-0324-X

Chaput, J. P., Gray, C. E., Poitras, V. J., Carson, V., Gruber, R., Birken, C. S... Tremblay, M. S. (2017). Systematic review of the relationships between sleep duration and health indicators in the early years (0-4 years). BMC Public Health, 17(5), 855. https://doi.org/10.1186/s12889-017-4850-2

Cohen, J. (1988). Statistical power analysis for the Behavioral Sciences. Hilsdale: Erlbaum.

Crabtree, V. M., Korhonenb, J. B., Montgomery-Downs, H. E., Jones, V. F., O'Brien, L. M. \& Gozala, D. (2005). Cultural influences on the bedtime behaviors of young children. Sleep Medicine, 6(1), 319-324. https://doi.org/10.1016/i. sleep.2005.02.001

Didden, R., Sigafoos, J. \& Lancioni, G. E. (2011). Unmodified Extinction for Childhood Sleep Disturbance. In M. Perlis, M. Aloia, \& B. Kuhn (Orgs.), Behavioral Treatments for Sleep Disorders: A Comprehensive Primer of Behavioral Sleep Medicine Interventions (pp. 257-263). London: Elsevier.
Durand, V. M. (2008). When Children Don't Sleep Well. Oxford: University Press.

Ferber, R. (2006). Solve your child's sleep problems. New York: Fireside Edition.

Gruber, R., Carrey, N., Weiss, S. K., Frappier, J. Y., Rourke, L., Brouillette, R. T...Wise, M. S. (2014). Position statement on pediatric sleep for psychiatrists. J Can Acad Child Adolesc Psychiatry, 23(3), 174-195.

Hill, C. (2011). Practitioner Review: Effective treatment of behavioural insomnia in children, Journal of Child Psychology and Psychiatry, 52(7), 731-741.

Hirshkowitz, M., Whiton, K., Albert, S. M., Alessi, C., Bruni, O., Don Carlos, L... Hillard, P. J. A.(2015). National Sleep Foundation's sleep time duration recommendations: methodology and results summary. Sleep Health, 1(1), 40-43. https://doi.org/10.1016/i.sleh.2014.12.010

Iglowstein, I., Jenni, O. G., Molinari, L., \& Largo, R. H. (2003). Sleep Duration From Infancy to Adolescence: Reference Values and Generational Trends. Pediatrics, 111(2), 302-307.

Khan, M. K. A., Chu, Y. L., Kirk, S. F. L., \& Veugelers, P. J. (2015). Are sleep duration and sleep quality associated with diet quality, physical activity, and body weight status? A population-based study of Canadian children. Can J Public Health, 106(5), e277-82. https://doi. org/10.17269/cjph.106.4892.

Kuhn, B. R. (2011). Behavioral Protocol to Promote Independent Sleep Initiation Skills and Reduce Bedtime Problems in Young Children. In M. Perlis, M. Aloia \& B. Kuhn (Orgs.), Behavioral Treatments for Sleep Disorders: A Comprehensive Primer of Behavioral Sleep Medicine Interventions (pp. 299-309). London: Elsevier.

Meltzer, L. J. \& Mindell, J. A. (2011). Graduated Extinction: Behavioral Treatment for Bedtime Problems and Night Wakings in Young Children. In M. Perlis, M. Aloia \& B. Kuhn. (Orgs.), Behavioral Treatments for Sleep Disorders: A Comprehensive Primer of Behavioral Sleep Medicine Interventions (pp. 265-273). London: Elsevier.

Meltzer, L. J. \& Mindell, J. A. (2014). Systematic Review and Meta-Analysis of Behavioral Interventions for Pediatric Insomnia. Journal of Pediatric Psychology, 39(8), 932-948. https://doi.org/10.1093/jpepsy/jsu041

Mindell, J. A., Kuhn, B., Lewin, D. S., Meltzer, L. J., \& Sadeh, A. (2006). Behavioral treatment of bedtime problems and night wakings in infants and young children - an American academy of sleep medicine review. Sleep, 29(1), 1263-76.

Mindell, J. A., Meltzer, L. J., Carskadon, M. A., \& Chervin, R. D. (2009a). Developmental aspects of sleep hygiene: Findings from the 2004 National Sleep Foundation Sleep in America Poll. Sleep Medicine, 10(1), 771-779. https://doi.org/10.1016/j.sleep.2008.07.016

Mindell, J. A. \& Moore, M. (2014). Bedtime Problems and Night Wakings. In S. H. Sheldon, R., Ferber, M. H., Kryger, \& D. Gozal (Orgs.), Principles \& Practice of Pediatric Sleep Medicine, (pp. 105-110). London: Elsevier. 
Mindell, J. A., Mond, C. E., Sadeh, A., Telofski, L., Kulkarni, N., \& Gunn, E. (2011). Efficacy of an Internet-Based Intervention for Infant and Toddler Sleep Disturbances. Sleep, 34(4), 451-458.

Mindell, J. A., Sadeh, A., Kohyama, J., \& How, T. H. (2010). Parental behaviors and sleep outcomes in infants and toddlers: A cross-cultural comparison. Sleep Medicine, 11(1), 393-399. https://doi.org/10.1016/i.sleep.2009.11.011

Mindell, J. A., Telofski, L. S., Wiegand, B., \& Kurtz, E. S. (2009). A Nightly Bedtime Routine: Impact on Sleep in Young Children and Maternal Mood. Sleep, 32(5), 599-606.

Mistry, K. B., Minkovitz, C. S., Strobino, D. M., \& Borzekowski, D. L. G. (2007). Children's Television Exposure and Behavioral and Social Outcomes at 5.5 Years: Does Timing of Exposure Matter? Pediatrics, 120(4), 762-769. https://doi.org/10.1542/peds.2006-3573

Moore, B. A, Friman, P. C., Fruzzetti, A. E., \& Macaleese, K. (2007). Brief report: evaluating the bedtime pass program for child resistance to bedtime d A randomized, controlled trial. Journal of Pediatric Psychology, 32, 283-287.

Moreira, G. A. \& Pradella-Hallinan, M. (2017). Sleepiness in Children: An Update. Sleep Medicine Clinic,12(3), 407413. https://doi.org/10.1016/j.jsmc.2017.03.013

Foundation National Sleep. (2016). How much sleep do we really need? Recuperado de https://Www sleepfoundation.org/how-sleep-works/how-much-sleep-do-we-really-need

Owens, J. A. (2007). Classification and epidemiology of childhood sleep disorders. Sleep Medicine Clinics, 2(1), 353-361. https://doi.org/10.1016/j.pop.2008.06.003

Pires, M. L. N., Vilela, C. B., \& Câmara, R. L. (2012). Desenvolvimento de uma medida de hábitos de sono e aspectos da prevalência de problemas comportamentais de sono na infância: uma contribuição. In N. S. Filho, D. P. S. A. Ribeiro, \& H. R. Rosa (Orgs.), Processos Clínicos e Saúde Mental (pp. 167-192). São Paulo: Cultura Acadêmica Editora \& Vetor Editora.

Rafihi-Ferreira, R. R., Pires, M. L. N., \& Silvares, E. F. M. (2019). Behavioral intervention for sleep problems in childhood: a Brazilian randomized controlled trial.Psicologia: Reflexão e Critica, 32(5). https://doi.org/10.1186/s41155-019-0118-3

Rafihi-Ferreira, R., Silvares, E. F. M., Pires, M. L. N., Assumpção-Jr, F. B., \& Moura, C. B. (2016). Sono e Comportamento em Crianças Atendidas em um Serviço de Psicologia. Psicologia: Teoria e Prática, 18(1), 159-172. https://doi.org/10.15348/1980-6906

Reid, G. J., Huntley, E. D., \& Lewin, D. S. (2009). Insomnias of Childhood and Adolescence. Child and Adolescent Psychiatric Clinics of North America, 18(1), 979-1000. https://doi.org/10.1016/j.chc.2009.06.002

Sadeh, A., Tikotzky, L., \& Scher, A. (2010). Parenting and infant sleep. Sleep Medicine Reviews, 14(1), 89-96. https://doi.org/10.1016/j.smrv.2009.05.003

Salti, R., Tarquini, R., Stagi, S., Perfetto, F., Cornélissen, G., Laffi, G., Mazzoccoli, G., \& Halberg, F. (2006). Age-dependent association of exposure to television screen with children's urinary melatonin excretion? Neuroendocrinology Letters, 27(1), 73-80.
Scharf, R. J., Demmer, R. T., Silver, E. J., \& Stein, R. E. (2013). Nighttime sleep duration and externalizing behaviors of preschool children. J Dev BehavPediatr, 34(6), 384-391. https://doi.org/10.1097/DBP.0b013e31829a7aod

Seymour, F. W., Bayfield, G., Brock, P., \& During, M. (1983). Management of night-waking in young children. Australian and New Zealand Journal of Family Therapy, 4, 217-223.

Sheldon, S. H. (2014). Development of Sleep in Infants and Children. In: S. H. Sheldon, R. Ferber, M. H. Kryger, \& D. Gozal (Orgs.), Principles \& Practice of Pediatric Sleep Medicine (pp. 17-24). London: Elsevier.

Short, M. A., Blunden, S., Rigney, G., Matricciani, L., Coussens, S., M. Reynolds, C., \& Galland, B. (2018). Cognition and objectively measured sleep duration in children: a systematic review and meta-analysis. Sleep Health, 4(3), 292-300. https://doi.org/10.1016/j.sleh.2018.02.004

Thomas, J. H., Moore, M., \& Mindell, J. A. (2014). Controversies in Behavioral Treatment of Sleep Problems in Young Children. Sleep Medicine Clinic, 9, 251-259. https://doi.org/10.1016/j.jsmc.2014.02.004

\section{Renatha El Rafihi-Ferreira}

Doutora em Psicologia Clínica pela Universidade de São Paulo (USP), em São Paulo, SP, Brasil; pesquisadora colaboradora no Instituto de Psiquiatria do Hospital das Clínicas da Faculdade de Medicina de São Paulo (IPq-HCFMUSP), em São Paulo, SP, Brasil. Bolsista FAPESP (2019/25537-3)

\section{Maria Laura Nogueira Pires}

Doutora em Ciências pela Universidade Federal de São Paulo (UNIFESP), em São Paulo, SP, Brasil; professora universitária aposentada da Universidade Estadual Paulista "Julio de Mesquita Filho" (UNESP), em Assis, SP, Brasil.

\section{Edwiges Ferreira de Mattos Silvares}

Doutora Livre Docente em Psicologia pela Universidade de São Paulo (USP), em São Paulo, SP, Brasil; professora universitária aposentada da Universidade de São Paulo (USP), em São Paulo, SP, Brasil.

\section{Endereço para correspondência}

Renatha El Rafihi-Ferreira

Instituto de Psiquiatria do Hospital das Clínicas da Faculdade de Medicina de São Paulo

R. Dr. Ovidio Pires de Campos, 785

05403-903

São Paulo, SP, Brasil 